\title{
Efficacy of Ronidazole for Treatment of Feline Tritrichomonas foetus Infection
}

\author{
Jody L. Gookin, Christina N. Copple, Mark G. Papich, Matthew F. Poore, Stephen H. Stauffer, \\ Adam J. Birkenheuer, David C. Twedt, and Michael G. Levy
}

\begin{abstract}
Objectives: To determine the efficacy of ronidazole (RDZ), tinidazole (TDZ), and metronidazole (MDZ) against Tritrichomonas foetus in vitro and of RDZ for treatment of feline naturally occurring or experimentally induced $T$ foetus infection.

Animals: A cat naturally infected with $T$ foetus infection and diarrhea. Ten specific-pathogen-free (SPF) kittens.

Procedure: RDZ, TDZ, and MDZ were tested for activity against 3 different feline isolates of $T$ foetus in vitro. RDZ then was administered to a naturally infected cat at $10 \mathrm{mg} / \mathrm{kg}$ PO q24h for 10 days. SPF kittens were infected orogastrically with feline $T$ foetus and treated with either placebo or RDZ (10 mg/kg PO q12h for 14 days). Cats with relapsing infection or those receiving placebo were treated subsequently with RDZ (either 30 or $50 \mathrm{mg} / \mathrm{kg}$ PO q12h for 14 days). Feces were examined for $T$ foetus by direct microscopy, culture, and polymerase chain reaction (PCR) testing weekly.

Results: Both RDZ and TDZ killed $T$ foetus at concentrations $>0.1 \mu \mathrm{g} / \mathrm{mL}$ in vitro. In the naturally infected cat, RDZ abolished diarrhea and $T$ foetus infection for 85 days after treatment, at which time infection and diarrhea relapsed. Retreatment with RDZ eradicated diarrhea and $T$ foetus infection for over 407 days. In experimentally induced infection, $\mathrm{RDZ}$ at $10 \mathrm{mg} / \mathrm{kg}$ caused initial improvement, but infection relapsed in all 5 cats 2 to 20 weeks after treatment. At 30 or $50 \mathrm{mg} / \mathrm{kg}, 10 / 10$ cats were negative for $T$ foetus infection for follow-up durations of 21 to 30 weeks after treatment.

Conclusions and Clinical Relevance: Oral administration of RDZ at 30 to $50 \mathrm{mg} / \mathrm{kg} \mathrm{q} 12 \mathrm{~h}$ for 14 days resolved diarrhea and eradicated infection (on the basis of polymerase chain reaction [PCR] testing) in 1 naturally infected cat and 10 experimentally inoculated cats receiving a different isolate of $T$ foetus.
\end{abstract}

Key words: Colitis; Diarrhea; Metronidazole; Nitroimidazole; Tinidazole.

$\mathbf{T}$ ritrichomonas foetus is a flagellated protozoan parasite that is best known as a venereal pathogen of cattle. Recently, $T$ foetus has been identified as an enteric pathogen of domestic cats that resides within the lumen of the colon and results in colitis and chronic, foul-smelling diarrhea. ${ }^{1-6}$ The origin of the feline infection is unknown. The infection is spread in cats via the fecal-oral route, and dense housing is an identified risk factor for infection. ${ }^{1-3} T$ foetus infection among domestic cats in catteries has become widespread; 36/117 $(31 \%)$ cats attending an international cat show were found to be infected with $T$ foetus, and infection was significantly associated with a history of diarrheal disease within the cattery. ${ }^{5}$ An effective treatment for $T$ foetus infection has yet to be identified; infected cats may have persistent diarrhea for up to 2 years, and some can remain infected for life. ${ }^{7}$ Trichomonads lack the ability to synthesize many essential macromolecules, which must be acquired from host secretions or by phagocytosis of host bacterial flora. Trichomonads also lack the mitochondria needed for aerobic metabolism.

From the Department of Molecular Biomedical Sciences (Gookin, Copple, Papich, and Stauffer ), Population Health and Pathobiology (Poore and Levy), and Clinical Sciences (Birkenheuer), College of Veterinary Medicine, North Carolina State University, Raleigh, NC; and the Department of Clinical Sciences, College of Veterinary Medicine and Biomedical Sciences, Colorado State University, Fort Collins, $C O$ (Twedt).

Reprint requests: Jody L. Gookin, Assistant Professor of Physiology and Internal Medicine, Department of Molecular Biomedical Sciences, College of Veterinary Medicine, North Carolina State University, 4700 Hillsborough Street, Raleigh, NC 27606; e-mail: Jody_Gookin@ncsu.edu.

Submitted June 5, 2005; Revised September 20, 2005; Accepted November 14, 2005.

Copyright $@ 2006$ by the American College of Veterinary Internal Medicine

0891-6640/06/2003-0010/\$3.00/0
Instead, pyruvate is generated by glycolysis and fermented in reductive organelles called hydrogenosomes. ${ }^{8}$ This reductive metabolic pathway serves as the basis for the susceptibility of trichomonads to 5nitroimidazole antibiotics, such as metronidazole $\left(\mathrm{MDZ}^{8}\right)$. Intact $T$ foetus reduce nitroimidazoles in hydrogenosomes, which results in generation of polar autotoxic anion radicals. ${ }^{9}$ Feline $T$ foetus infection has not been responsive to MDZ, commonly used in veterinary practice. Cats have shown transient improvement in diarrhea while receiving MDZ but remain infected with the organism. ${ }^{1,7}$ Because of the lack of success with MDZ, it was necessary to investigate other compounds having a similar mechanism of action but potentially greater activity against $T$ foetus.

The specific aims of the present study were (1) to determine the susceptibility of feline $T$ foetus to each of 3 nitroimidazole antimicrobials (ronidazole [RDZ], tinidazole [TDZ], and MDZ) in vitro and (2) to determine the ability of RDZ to resolve diarrhea and eradicate $T$ foetus infection, on the basis of polymerase chain reaction (PCR) testing, when given orally to cats with either a natural or experimentally induced infection.

\section{Materials and Methods}

\section{Isolates}

Three different isolates of $T$ foetus were examined for their susceptibility to RDZ, TDZ, and MDZ in vitro. Isolate 1 was derived from a naturally infected cat in a North Carolina shelter. Isolate 2 was derived from the naturally infected cat treated with $\mathrm{RDZ}$ in this study. Isolate 3 was derived from a cat performing in a cat show in Houston, Texas. This latter isolate was used for experimental inoculations. Each isolate was established in antibiotic-free culture medium as previously described ${ }^{2}$ and positively identified as $T$ foetus on the basis of single-tube nested PCR testing for detection of the ribosomal RNA (rRNA) gene unit using species-specific primers. 


\section{In Vitro Susceptibility Assay}

Feline $T$ foetus in log-phase culture were inoculated at a concentration of $10^{3}$ organisms $/ \mathrm{mL}$ into $10-\mathrm{mL}$ culture tubes containing culture medium ${ }^{\mathrm{a}}$ supplemented with $\mathrm{RDZ}^{\mathrm{b}}$ or $\mathrm{TDZ}^{\mathrm{c}}$ at concentrations of $0,0.01,0.1,1$, and $10 \mu \mathrm{g} / \mathrm{mL}$ ( 3 replicates each) and incubated at $37^{\circ} \mathrm{C}$. For comparison, MDZ was tested at concentrations of 0,1 , and $10 \mu \mathrm{g} / \mathrm{mL}$. At each concentration studied, the powder formulation of the respective nitroimidazole was dissolved directly into a volume of culture medium sufficient for aliquoting into 3 replicate tubes.

After 24, 48, and 72 hours of incubation, organisms were suspended by lightly vortexing, and then $0.1 \mathrm{~mL}$ of the suspended culture was diluted and fixed in $0.9 \mathrm{~mL}$ of $10 \%$ formaldehyde. Cell counts were performed using a hemocytometer and reported as the mean $\pm \mathrm{SD}$ of each triplicate dilution. At 72 hours, 1 tube at each dilution was centrifuged, and then $0.1 \mathrm{~mL}$ of pelleted organisms was inoculated into $10 \mathrm{~mL}$ of antibiotic-free medium. At 24 hours, a $10 \mu \mathrm{L}$ aliquot of each subculture was examined by light microscopy for motile trichomonads.

The entire in vitro susceptibility assay was performed on each of 3 different $T$ foetus isolates. Susceptibility of $T$ foetus to a given concentration of drug was defined by the absence of replication over a 72-hour period and the failure of the organisms to replicate after transfer to antibiotic-free media.

\section{Cat with Naturally Occurring Infection}

A 3-year-old spayed female Persian cat was donated to North Carolina State University at 1 year of age because of a 6-month history of unrelenting large bowel diarrhea. Infection with $T$ foetus was confirmed by direct microscopy, microbial culture for trichomonads, ${ }^{\mathrm{d}, 10}$ and single-tube nested PCR testing for detection of the rRNA gene unit of $T$ foetus. ${ }^{11}$ The cat was determined to be otherwise healthy on the basis of physical examination, serum biochemistry profile, preprandial and postprandial serum bile acid concentrations, feline leukemia and feline immunodeficiency virus testing, and urinalysis results. Fecal examinations also included flotation for parasite ova in saturated sodium nitrate (specific gravity 1.200); direct microscopy of a hanging drop for motile spiral organisms, such as Helicobacter and Campylobacter spp; Gram stain and antigen testing for Giardia spp. ${ }^{e}$ While housed at North Carolina State University, the cat was treated with a variety of different antimicrobials, none of which were effective in eradicating the $T$ foetus infection. The cat was eventually adopted and housed alone in an outdoor facility. For the 2-year period before the present study, diarrhea remained unrelenting, often contained fresh blood and mucus, and frequently dribbled from the rectum.

A pure culture of $T$ foetus was derived from the cat's feces by a method previously described, ${ }^{3}$ and the organisms were tested in vitro for susceptibility to RDZ, TDZ, and MDZ. The cat then was treated with RDZ at a dosage of $10 \mathrm{mg} / \mathrm{kg}$ of body weight PO q24h for 10 days. $\mathrm{CBC}$, serum biochemistry profile, and urine analyses were performed immediately before and after completion of treatment. Fecal samples were collected at varying time intervals after treatment and tested with a single-tube nested PCR assay for $T$ foetus. Fecal consistency was noted periodically.

\section{Experimentally Induced Infection}

Ten specific-pathogen-free (SPF) 10-week-old sexually intact female domestic shorthair cats were purchased from a commercial vendor. ${ }^{f}$ The cats were randomly divided into 2 equal groups. Each group was housed in a separate room, and all cats in each room were kept in separate cages and received the same dry food ${ }^{\mathrm{g}}$ ad libitum throughout the study. Each day, the cage liner was scraped clean and a new litter box was provided. Cats were transferred to freshly disinfected cages once per week. Cats were housed under conditions of controlled lighting and temperature and were maintained in compliance with biosafety-level-2 guidelines. The North Carolina State University Institutional Animal Care and Use Committee approved all protocols.

During an initial 3-week acclimatization period, each cat was determined to be healthy on the basis of physical examination, $\mathrm{CBC}$, serum biochemistry profile, and urinalysis results. Fecal examination for enteric pathogens included a flotation in saturated sodium nitrate (specific gravity 1.200) for parasite ova, direct microscopy for protozoa $(n=3$ examinations/cat $)$ and motile spiral organisms, microbial culture for trichomonas ${ }^{\mathrm{d}, 10}$ (3 examinations/ cat), antigen testing for Giardia spp, ${ }^{\mathrm{e}}$ and single-tube nested PCR testing for detection of the rRNA gene unit of $T$ foetus ${ }^{11}$ (3 examinations/cat).

\section{Inoculation with $\mathrm{T}$ foetus}

Trichomonads were isolated from a naturally infected cat, grown in culture as previously described, ${ }^{3}$ and cryopreserved at $-70^{\circ} \mathrm{C}$. The organisms were thawed on ice and passed 5 times during a 2-week period before use for experimental inoculation. The final passage was established in antibiotic-free modified Diamond media, ${ }^{a}$ and the concentration of trichomonads was quantified by means of a hemocytometer. Each cat received $3.3 \mathrm{~mL}$ of media containing approximately $3 \times 10^{6}$ live $T$ foetus via orogastric intubation. On the basis of PCR amplification and sequencing, the isolate used for experimental inoculation had $100 \%$ sequence identity with the rRNA gene sequence of $T$ foetus (GenBank No. AF466749).

\section{Treatment of Experimentally Induced Infection}

Treatment with RDZ was initiated 4 weeks after the experimentally induced infection; a time period corresponding to observation of diarrhea in $100 \%$ of experimentally infected cats in a previous study. ${ }^{3}$ At that time, each group of cats $(n=5$ each) was blindly allocated to administration of treatment or placebo. The treatment group received RDZ [(1-methyl-5-nitroimidazole-2yl)-methyl carbamate] ${ }^{\mathrm{b}}$ at a dosage of $10 \mathrm{mg} / \mathrm{kg}$ of body weight $\mathrm{PO}$ q12h. The placebo group received an equal weight of dextrose PO $\mathrm{q} 12 \mathrm{~h}$. Both compounds were obtained from a commercial supplier ${ }^{\mathrm{b}}$ and compounded into opaque, color-coded gel capsules by the College of Veterinary Medicine pharmacy. Each compound was administered for 14 days.

\section{Duration of Follow-up}

Feces from each cat were tested for the presence of $T$ foetus on the basis of PCR testing once a week for a minimum of 6 weeks. This minimum follow-up period was originally selected based on our prior observation that the PCR assay had a sensitivity of $100 \%$ when $\geq 4$ consecutive fecal samples were tested in a prior study. ${ }^{11}$ Additionally, residual antibiotic theoretically could result in falsenegative test results by suppressing but not eradicating $T$ foetus. Thus, we conservatively estimated that residual antibiotic should be negligible after 2 weeks. After the original 6-week follow-up period, cats failing treatment at low dosages of RDZ began treatment at a higher dosage. Meanwhile, the duration of follow-up for the remaining cats was extended, resulting in recognition that cats could relapse as long as 20 weeks after completion of treatment. Thus, cats subsequently treated with 30 or $50 \mathrm{mg} / \mathrm{kg}$ of RDZ were evaluated weekly by PCR testing for a minimum of 20 weeks.

\section{Disposition of Treatment Failures}

Upon completion of the blinded study, when the cats in the treatment group had a relapsing infection or if they originally 
received placebo, they were treated with $\mathrm{RDZ}$ at a higher dosage of either $30 \mathrm{mg} / \mathrm{kg}(\mathrm{n}=3)$ or $50 \mathrm{mg} / \mathrm{kg}(\mathrm{n}=7)$ body weight PO q12h for 14 days. Cats were tested for $T$ foetus infection weekly over a minimum of 21 weeks after the last dose of RDZ. Cats receiving $10 \mathrm{mg} / \mathrm{kg} \mathrm{RDZ}$ and failing to clear $T$ foetus had their organisms reisolated in culture and tested in vitro for susceptibility to RDZ (range 0.01 to $1000 \mu \mathrm{g} / \mathrm{mL}$ ) followed by subculture in antibioticfree media as previously described.

\section{Data Collection}

For cats with experimentally induced infection, fecal consistency was recorded daily on the basis of a previously published 4-point scale (1, formed [normal consistency and hard, with distinct or impacted fecal balls]; 2, semiformed [voluminous, smooth-surfaced, and soft; discrete fecal balls not evident]; 3 , cow pie [not formed, consisting of loose puddles and piles of wet feces]; and 4, liquid [watery and loose]..$^{3}$ Also recorded daily were the total number of bowel movements, number of episodes of vomiting, and a subjective assessment of each cat's appetite and mentation (normal versus abnormal). A complete physical examination was performed once weekly.

Fecal examinations for $T$ foetus were performed on all cats weekly over a 52-week period. Feces from each cat were collected from the rectum with a plastic loop and immediately processed for examination. Analysis of fecal samples for $T$ foetus included microscopic examination of a fecal smear, culture of feces in a commercially available culture system, ${ }^{\mathrm{d}}$ and single-tube nested PCR testing for detection of the $T$ foetus rRNA gene unit using species-specific primers. These techniques have been previously described in detail. ${ }^{10,11}$

Before PCR testing for $T$ foetus, all samples of DNA extracted from feces were first subjected to PCR amplification of an 876 base pair (bp) gene sequence of bacterial 16S rRNA. Reaction conditions for 16S rRNA gene amplification were as follows: a 100$\mu 1$ reaction volume of PCR buffer II containing $2.5 \mathrm{U}$ AmpliTaq Gold DNA polymerase, ${ }^{\text {h }} 100$ pmol each of primers $515 \mathrm{~F}\left(5^{\prime}\right.$ GTGCCAGCAGCCGCGGTAA 3') and 1391R (5' GACGGGCGGTGAGTGCA $3^{\prime}$ ), $200 \mu \mathrm{M}$ each deoxynucleotide triphosphate, $10 \mu \mathrm{g}$ of bovine serum albumin, and $5 \mu \mathrm{L}$ of DNA template. DNA amplification was performed at the following temperature profiles: initial denaturation at $95^{\circ} \mathrm{C}$ for 5 minutes, denaturation at $95^{\circ} \mathrm{C}$ for 1 minute, annealing at $48^{\circ} \mathrm{C}$ for 1 minute, and extension at $72^{\circ} \mathrm{C}$ for 2 minutes for 50 cycles, followed by a final extension for 5 minutes at $72^{\circ} \mathrm{C}$. By performing PCR testing for bacterial 16S rRNA gene, the possibility that a negative PCR test result for $T$ foetus could be attributed to the presence of endogenous PCR inhibitors in the extracted DNA was ruled out for each sample.

For evaluation of adverse acute systemic effects, CBC, serum biochemistry profile, and urine analyses were performed on all cats immediately before experimental inoculation with $T$ foetus and within a week after completion of treatment with placebo or RDZ $(10 \mathrm{mg} / \mathrm{kg})$. Prior studies with this model have shown that infection alone does not result in hematologic or serum biochemical abnormalities. ${ }^{3}$ In addition, all 7 cats treated with RDZ at $50 \mathrm{mg} / \mathrm{kg}$ had CBC, serum biochemistry profile, and urine analyses performed immediately before and within a week after completing treatment.

\section{Statistical Analysis}

All statistical analyses were performed using a commercial software package. ${ }^{i}$ To determine whether $T$ foetus infection significantly increased fecal consistency scores or defecation frequency compared to the pre-infection period, the daily fecal consistency score and number of bowel movements of each individual cat were each averaged over the 2 -week period before inoculation with $T$ foetus and over the 2-week period after inoculation with $T$ foetus. Whether there was a difference in average fecal consistency score or defecation frequency of cats between the pre-infection and postinfection periods was tested using a Student's paired $t$-test. For all analyses, values of $P<.05$ were considered significant.

\section{Results}

\section{In Vitro Susceptibility of $\mathrm{T}$ foetus to 5-Nitroimidazoles}

There was no inhibitory effect with MDZ on proliferation of $T$ foetus in vitro at concentrations $\leq 10 \mu \mathrm{g} / \mathrm{mL}$. Both TDZ and RDZ arrested growth of $T$ foetus in vitro at concentrations $\geq 0.1 \mu \mathrm{g} / \mathrm{mL}$. At a concentration of $0.01 \mu \mathrm{g} / \mathrm{mL}$, more sustained attenuation of growth was observed with RDZ (Fig 1). Trichomonads did not replicate after transfer from tubes containing $\geq 0.1 \mu \mathrm{g} / \mathrm{mL}$ TDZ or RDZ to antibiotic-free media.

\section{Effect of RDZ on Naturally Occurring Infection in a Cat}

Within 24 hours of initiating treatment with RDZ at $10 \mathrm{mg} / \mathrm{kg}$ PO q24h, trichomonads were no longer detected by direct examination of fecal smears. Improvement in fecal consistency was immediate and dramatic, with feces returning to normal after completion of the 10-day treatment course (Fig 2). No adverse effects, such as vomiting or inappetence, were observed during or after treatment. Results of $\mathrm{CBC}$, serum biochemistry profile, and urine analyses remained within reference range limits. Feces remained formed and tested negative for $T$ foetus infection by culture and single-tube nested PCR assay on days 15 and 31 after treatment. On day 85 after treatment, there was an acute onset of large bowel diarrhea containing fresh blood and mucus. Feces were again positive for $T$ foetus infection by direct smear examination, culture, and single-tube nested PCR testing (Fig 2). Diarrhea was so severe that perineal soiling with feces resulted in infestation by maggots, which was treated by local debridement, wet-to-dry bandaging, and administration of systemic antibiotics.

$T$ foetus was re-isolated from the cat's feces and was determined in vitro to remain susceptible to killing by RDZ at concentrations $\geq 0.1 \mu \mathrm{g} / \mathrm{mL}$. The cat was retreated with RDZ at the same dosage and duration (10 $\mathrm{mg} / \mathrm{kg}$ body weight PO q24h for 10 days). Immediate resolution of diarrhea and fecal shedding of trichomonads again was observed. Feces remained formed, and the cat has tested negative for $T$ foetus infection by single-tube nested PCR assay on days 22, 37, and 407 after retreatment with RDZ (Fig 3).

\section{Experimentally Induced Infection with $\mathrm{T}$ foetus}

No cat tested positive for $T$ foetus before the experimentally induced infection. Feces from 1 cat contained small numbers of Isospora spp ova. Within 2 weeks of orogastric inoculation, feces from each cat tested positive for $T$ foetus infection. There was 

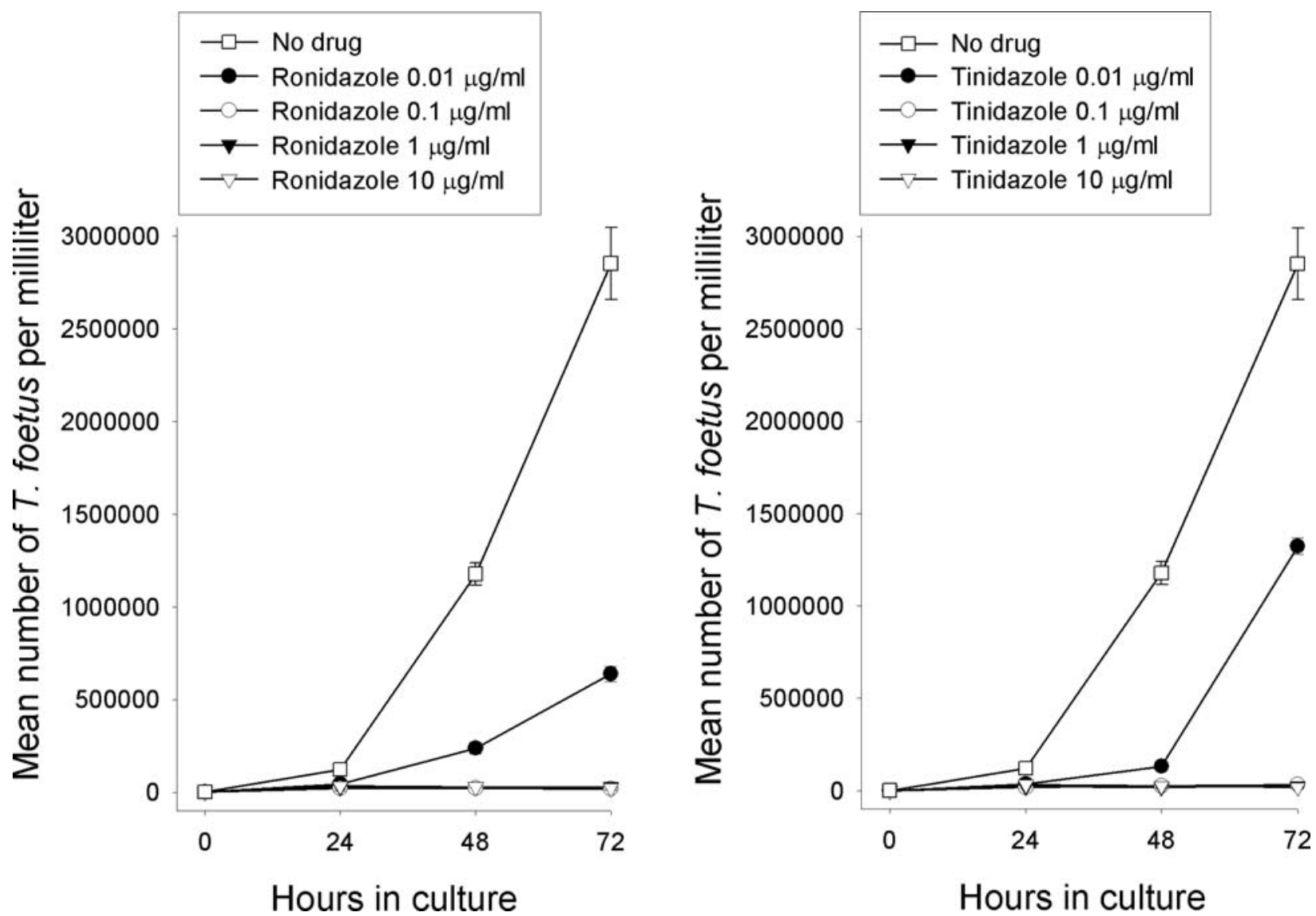

Fig 1. In vitro susceptibility of feline Tritrichomonas foetus to increasing concentrations of ronidazole (RDZ) or tinidazole (TDZ). Data points represent the mean \pm SD of 3 replicates at each dilution. Data shown are from one isolate and are representative of 3 separate experiments, each performed with a different isolate of feline $T$ foetus.

a significant difference in the average fecal consistency of cats after infection compared to the pre-infection period $(P=.01$, Fig 3$)$. All cats developed semiformed to liquid diarrhea by the 2nd week of infection. In 6 cats, involuntary dribbling of feces also was observed (Fig 3). There was no difference in the frequency of defecation after infection with $T$ foetus (average number of bowel movements per day: $1.2 \pm 0.22$ before infection, $1.3 \pm$ 0.29 after infection $[\mathrm{n}=10, P=0.12])$. Despite persistent infection, severity of diarrhea began to abate in all cats before beginning treatment with RDZ (Fig 3). Therefore, it was not possible to statistically evaluate whether treatment with RDZ improved fecal consistency. Spontaneous resolution of $T$ foetus infection was not observed in any cat.

\section{Effect of RDZ on Cats with Experimentally Induced Infection}

All 5 cats in the treatment group receiving RDZ at $10 \mathrm{mg} / \mathrm{kg}$ of body weight PO q12h became negative for $T$ foetus infection (as determined by direct fecal smear, culture, and PCR testing) within 3 days of initiating treatment. These cats remained negative throughout the 2-week treatment period and for 1 week thereafter. However, a relapsing infection was detected at least once in each cat in the treatment group at 2, 3, 3, 17, or 20 weeks after the treatment course was completed (Fig 4). $T$ foetus was re-isolated from 1 of these cats' feces and was determined in vitro to remain susceptible to killing by RDZ at concentrations $\geq 0.1 \mu \mathrm{g} / \mathrm{mL}$. The 5 cats receiving placebo remained positive for $T$ foetus infection throughout this time.

Three treatment group cats (those relapsing at 2 or 3 weeks after treatment) were retreated with $\mathrm{RDZ}$ at $30 \mathrm{mg} / \mathrm{kg}$ of body weight PO $\mathrm{q} 12 \mathrm{~h}$ for 14 days. The remaining 2 treatment group cats (those relapsing at 17 and 20 weeks after treatment) and those originally treated with placebo $(n=5)$ were treated with $\mathrm{RDZ}$ at $50 \mathrm{mg} / \mathrm{kg}$ of body weight PO q12h for 14 days. Two cats receiving $30 \mathrm{mg} / \mathrm{kg}$ and all 7 cats receiving $50 \mathrm{mg} / \mathrm{kg}$ remained negative for $T$ foetus for follow-up durations of 30 and 21-23 weeks after treatment, respectively (Fig 4). The remaining cat that received $30 \mathrm{mg} / \mathrm{kg} \mathrm{RDZ}$ also was negative for $T$ foetus for 18 weeks after treatment, at which time the cat died acutely. Complete postmortem and histopathologic examinations did not identify a cause of death.

Adverse events (eg, lethargy, vomiting, anorexia) were not observed during or after treatment with RDZ. Subjectively, feces were softer while cats received $\mathrm{RDZ}$ at $50 \mathrm{mg} / \mathrm{kg}$ of body weight. Results of CBC, serum biochemistry profile and urine analyses performed before and immediately after cats received 

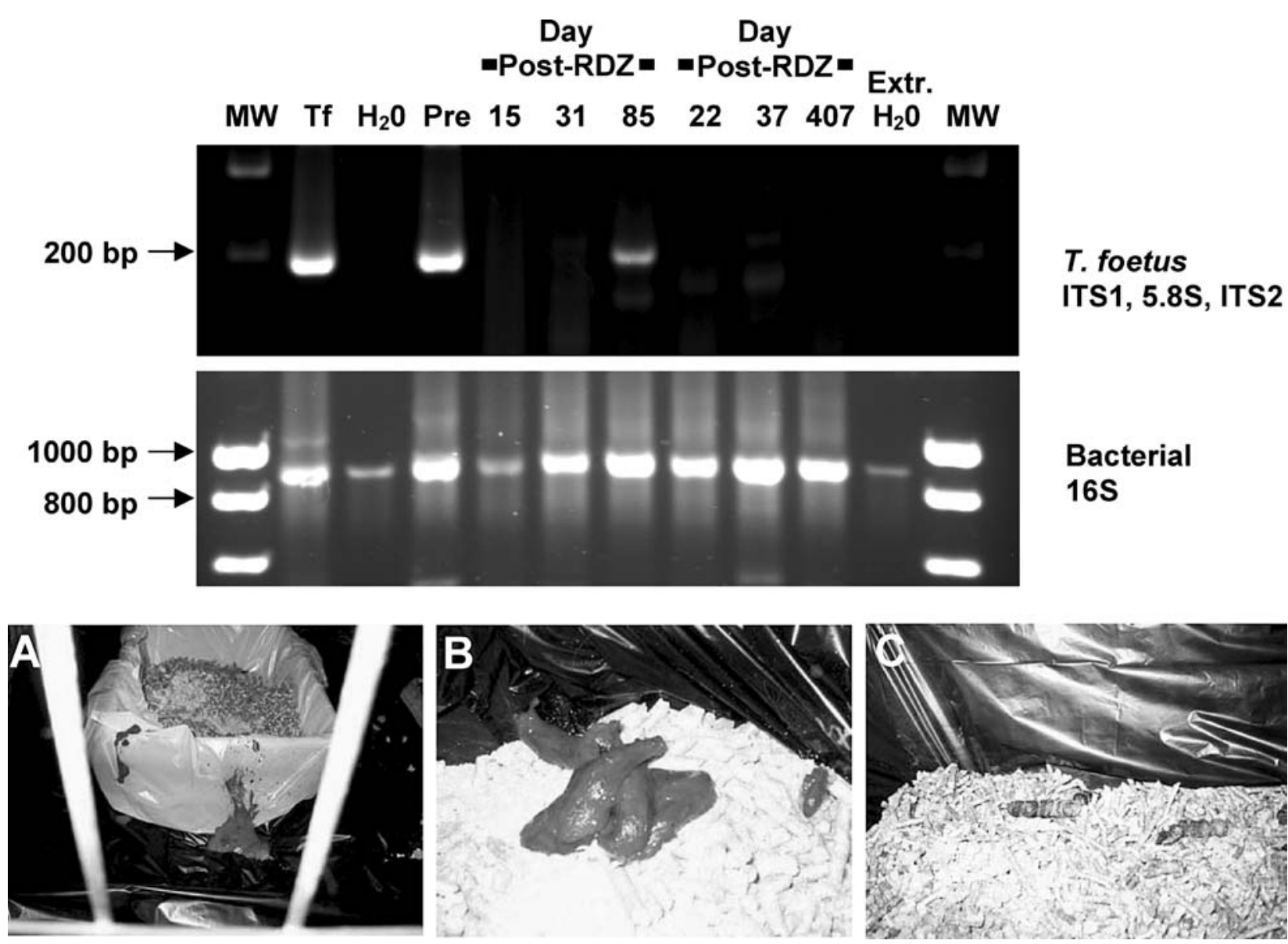

Fig 2. Analysis of single-tube nested polymerase chain reaction (PCR) amplification products of Tritrichomonas foetus partial rRNA gene unit by $1.5 \%$ agarose gel electrophoresis followed by staining with ethidium bromide. From left to right, lanes show the following: molecular weight markers; $T$ foetus genomic DNA, sterile water (PCR contamination control); DNA extracted from feces of a naturally infected cat before (day $0=$ pre) and after treatment with RDZ (day 15 and 31) and at the time of relapse infection (day 85); DNA extracted from feces of a naturally infected cat 22, 37, and 407 days after retreatment with RDZ; DNA extracted in the absence of feces (DNA extraction contamination control); and molecular weight markers. Analysis of bacterial 16S rRNA gene products in each sample ruled out the presence of endogenous PCR inhibitors. Appearance of voided feces from a cat with naturally occurring $T$ foetus infection: Panel A, before treatment with RDZ; Panel B, after 48 hours of treatment with RDZ; and Panel C, 2 months after completion of treatment with RDZ.

$\mathrm{RDZ}$ at $10 \mathrm{mg} / \mathrm{kg}$ or $50 \mathrm{mg} / \mathrm{kg}$ body weight remained within reference range limits. Because clinically relevant changes between pretreatment and posttreatment hematologic or biochemical data were not observed, statistical comparisons were not performed.

\section{Discussion}

An effective treatment for feline $T$ foetus infection or the associated diarrhea has not been reported previously. In infected cats, diarrhea typically waxes and wanes, often appearing to respond to treatment only to relapse shortly after treatment is discontinued. ${ }^{1} \mathrm{MDZ}$ often is chosen as initial therapy, either because trichomonads initially are mistaken for Giardia spp. or are thought to be MDZ-sensitive trichomonads, such as Pentatrichomonas hominis, or because no other proven treatment options exist. Our finding in the present study that MDZ had no activity against 3 different isolates of $T$ foetus in vitro is consistent with the observed lack of its clinical efficacy. ${ }^{1}$
In the present study, we tested 2 related 5-nitroimidazoles, TDZ and RDZ, for in vitro activity against feline $T$ foetus. TDZ is licensed for use in people for treatment of infections caused by MDZ-resistant Trichomonas vaginalis. RDZ has demonstrated activity against Histomonas meleagridis (turkey blackhead), Treponema hyodysenteriae (swine dysentery), and Trichomonas gallinae infection (pigeon canker). ${ }^{13-15} \mathrm{RDZ}$ is not registered for human or veterinary use in the United States, but chemical grade RDZ is commercially available. ${ }^{\mathrm{b}}$

In vitro, RDZ and TDZ demonstrated microbicidal activity against 3 different isolates of feline $T$ foetus. The activity of RDZ was slightly greater than that of TDZ. Thus, RDZ was chosen for evaluation in treating in vivo $T$ foetus infection. The dosage of RDZ for in vivo treatment of $T$ foetus-infected cats was extrapolated from the dosing recommendations for the related nitroimidazole MDZ, ${ }^{16}$ the dosage reported for use in pigeons ${ }_{15}^{15}$ and the results of empiric treatment of the naturally infected cat in this report. Although there are 

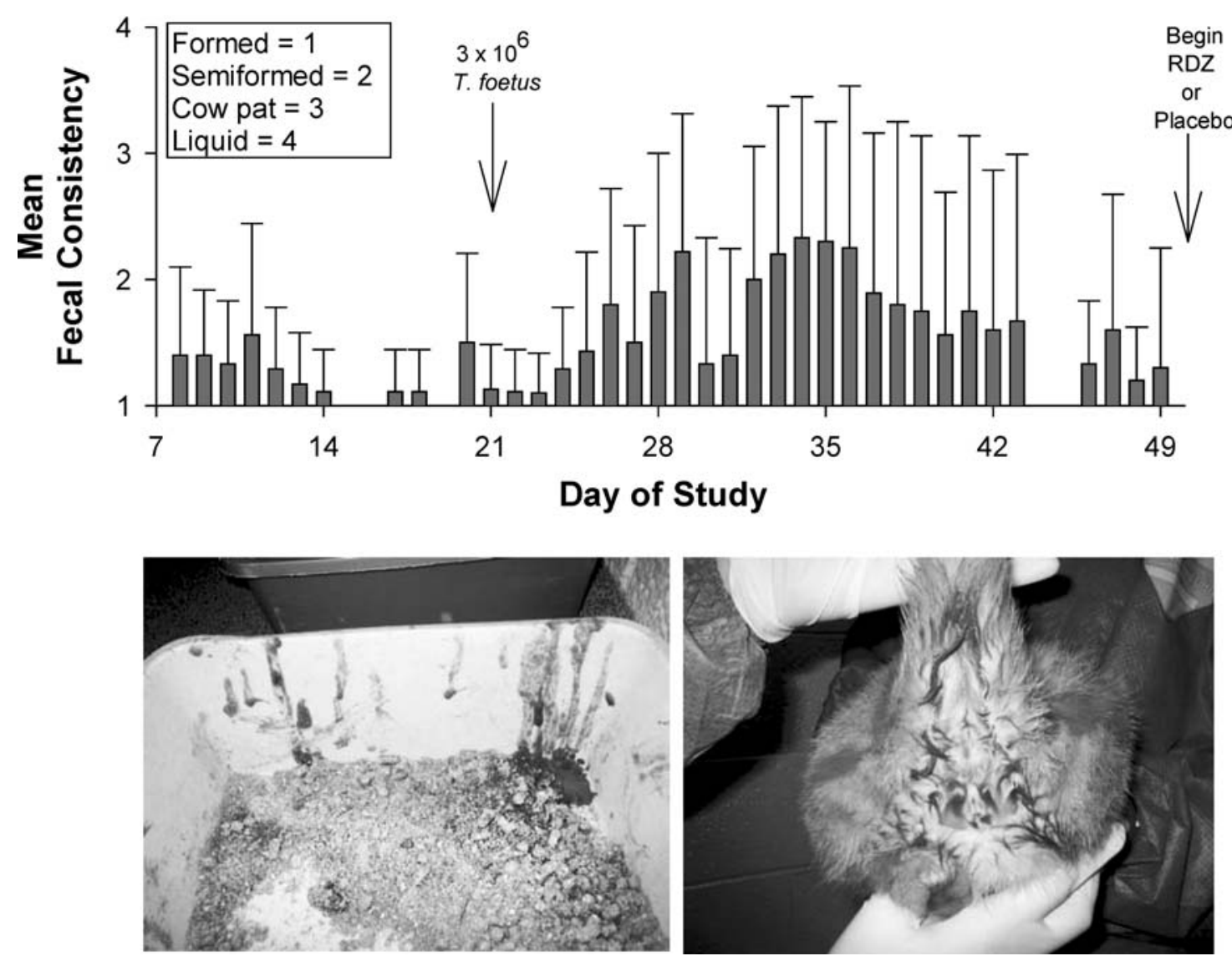

Fig 3. Daily mean ( \pm SD) fecal consistency score for 10 cats before and after experimental inoculation with Tritrichomonas foetus. Fecal consistency for each cat was scored daily as follows: 1, formed; 2 , semiformed; 3, cow pie; and 4, liquid. Ronidazole (RDZ) administered at $10 \mathrm{mg} / \mathrm{kg}$ PO q12h. Appearance of voided feces and perineal region of a cat with experimentally induced $T$ foetus infection. Note the severity of diarrhea (score $=4$ ) and the presence of fecal incontinence.

no pharmacokinetic or toxicity data on the use of RDZ in cats, RDZ has a wide therapeutic safety margin in birds. ${ }^{17}$ Furthermore, $T$ foetus has been shown to avidly metabolize RDZ by pathways different from those used by mammalian and gut bacterial enzymes, which may provide attributes of safety for RDZ compared to MDZ. ${ }^{18}$
In the present study, RDZ was well tolerated by all cats receiving dosages up to $50 \mathrm{mg} / \mathrm{kg}$ of body weight $\mathrm{q} 12 \mathrm{~h}$ for 14 days. When administered to a naturally infected cat, RDZ resulted in acute, marked, and sustained resolution of diarrhea, despite a prolonged history of unrelenting large bowel diarrhea. Unfortunately, we were unable to evaluate the effect of RDZ on

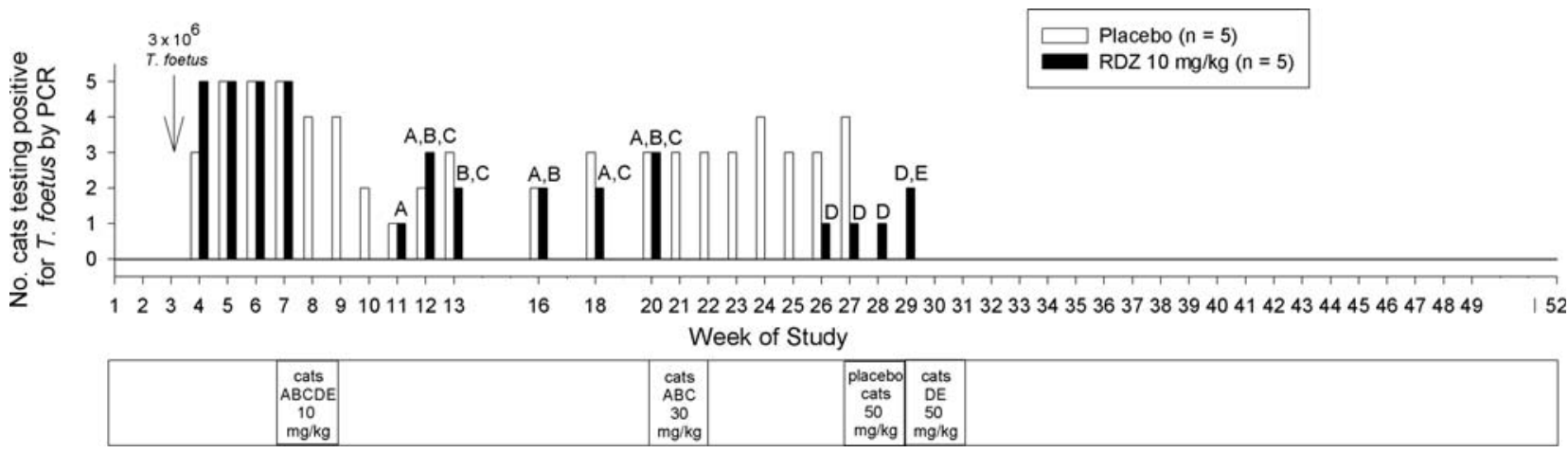

Fig 4. Time line of experimental feline Tritrichomonas foetus infection and response to treatment with ronidazole (RDZ). Ten specificpathogen-free cats were acclimated for 3 weeks before experimental infection with $T$ foetus (arrow). Before and after infection, feces from each cat was tested on the weeks shown for the presence of $T$ foetus by a single-tube nested PCR assay. Cats originally receiving $10 \mathrm{mg} / \mathrm{kg}$ $\mathrm{RDZ}$ are designated as A, B, C, D, and E. Onset and duration of each treatment period is shown beneath the x-axis, and indicates the dose administered and identity of the cats receiving each dose. 
diarrhea in experimentally infected cats because diarrhea began to spontaneously abate by the time treatment was initiated 4 weeks after infection. In response to treatment with RDZ, all cats became negative for $T$ foetus on the basis of direct smear, culture, and singletube nested PCR testing of fecal samples. However, infection relapsed in all cats receiving $\mathrm{RDZ}$ at a dosage of $10 \mathrm{mg} / \mathrm{kg}$ body weight. Relapse caused by suboptimal dosage rather than re-infection is considered more likely in these cats because all were housed separately and with strict attention to sanitation. $T$ foetus that was recultured from the feces of 1 cat with relapsing infection remained susceptible to killing by RDZ in vitro. At RDZ doses of 30 or $50 \mathrm{mg} / \mathrm{kg}$ body weight, all cats became negative for $T$ foetus on the basis of repeated PCR testing over durations of 21 to 30 weeks. We believe that the spontaneous remission of $T$ foetus infection in the cats reported here is unlikely. First, spontaneous remission of $T$ foetus infection was not observed in any cats in this study. Second, spontaneous remission was not observed in any cats over a period of $\geq 50$ weeks after experimental infection with $T$ foetus in a prior study. ${ }^{3}$ Finally, all cats became PCR negative for $T$ foetus infection within days of initiating treatment with RDZ.

Compared to MDZ, the greater efficacy of RDZ for treatment of cats with $T$ foetus infection may be attributed to a higher activity of RDZ in vivo and trapping of the activated compound within the intestine. RDZ has a 10-fold higher in vivo activity against trichomonal infections compared to MDZ. ${ }^{18}$ In pigs, a high fraction of RDZ is found in the feces, which may be the result of reductive activation of the compound and trapping of the reactive intermediate by anaerobic organisms in the intestinal tract. ${ }^{19}$

In the present study, a sensitive and specific PCR assay was used to repeatedly test the feces of cats for the presence of the rRNA genes of $T$ foetus. This PCR assay has been validated for use with DNA extracted from feline feces and is the only PCR assay superior to culture for demonstrating $T$ foetus organisms in biological samples., ${ }^{50-22}$ Feces is considered to be one of the most complex biological samples for PCR testing because of the presence of inherent PCR inhibitors, such as heme, bilirubin, bile salts, and complex carbohydrates. ${ }^{23}$ To minimize the possibility of false-negative results caused by the presence of PCR inhibitors, DNA extractions were performed by a protocol optimized for fecal samples, ${ }^{11}$ and every DNA sample was tested for PCR inhibitors by a separate reaction in which bacterial 16S rRNA genes were amplified before testing with $T$ foetus-specific primers. The $\sim 900$ bp 16S rRNA gene amplicon was frequently detected in negative control samples (Fig 2), which is consistent with the presence of low amounts of bacterial DNA in Taq polymerase. ${ }^{24}$ Because the purpose of this PCR test was to detect the presence of PCR inhibitors in each sample before testing for $T$ foetus, we decided not to pretreat the Taq polymerase with DNase I because this procedure has been associated with reduced sensitivity of subsequent PCR. ${ }^{24}$

Our findings suggest that RDZ at 30 to $50 \mathrm{mg} / \mathrm{kg}$ body weight $\mathrm{PO} \mathrm{q} 12 \mathrm{~h}$ for 14 days results in long-term elimination of $T$ foetus infection and diarrhea in the cat. Nevertheless, although PCR testing can be used to confirm infection, it cannot be used to conclusively prove that infection has been eradicated. In the present study, prolonged periods of asymptomatic, PCR-negative intervals were observed after initial therapy with RDZ. Our duration of follow-up was extended from 6 to $\geq 21$ weeks when we observed that infection could relapse as long as 20 weeks after treatment. Thus, shortterm studies of drug efficacy in $T$ foetus infected cats should be viewed with caution. In all cats that relapsed after initial treatment with RDZ, follow-up treatment with RDZ eradicated infection.

In the present study, 3 different strains of feline $T$ foetus were demonstrated to be susceptible to killing by RDZ. At least 1 of these strains is known to have been derived from a cat previously treated, without success, with multiple antimicrobial drugs. In practice, the efficacy of RDZ may be altered by variation in antimicrobial susceptibility among different field strains of feline $T$ foetus. Thus, clinical experience ultimately will be necessary to determine whether RDZ cures $T$ foetus infected cats and eliminates them as carriers for transmission.

\section{Footnotes}

\footnotetext{
${ }^{a}$ Modified Diamond medium, Remel, Lenexa, KS

${ }^{\mathrm{b}}$ Ronidazole [(1-methyl-5-nitroimidazole-2-yl)-methyl carbamate; $>99 \%$ pure] R7635-5G, Sigma Chemical Co, St. Louis, MO

${ }^{\mathrm{c}}$ Tinidazole [1-(2(ethylsulfonyl)ethyl)-2-methyl-5 nitroimidazole] T3021, Sigma Chemical Co, St. Louis, MO

${ }^{\mathrm{d}}$ In Pouch TF, Biomed Diagnostics Inc, White City, OR

${ }^{\mathrm{e}}$ ProSpecT Giardia Rapid Assay, Alexon-Trend Inc, Ramsey, MN

${ }^{\mathrm{f}}$ Liberty Research Inc, Waverly, NY

${ }^{g}$ Hills' Science Diet Feline Growth, Hills' Nutrition, Topeka, KS

${ }^{\text {h }}$ Perkin-Elmer, Foster City, CA

${ }^{i}$ SigmaStat statistical software, Jandel Scientific, San Rafael, CA
}

\section{Acknowledgments}

This work was funded by a state of North Carolina appropriated competitive research grant. An abstract of this work was presented at the American College of Veterinary Internal Medicine 23rd Annual Forum, Baltimore, MD, June 2005. The authors thank Drs Steve Weir and Kristin Lowrimore, Ms GiGi Davidson and Robin Gager, and the North Carolina State University College of Veterinary Medicine laboratory animal resources personnel.

\section{References}

1. Gookin JL, Breitschwerdt EB, Levy MG, et al. Diarrhea associated with trichomonosis in cats. J Am Vet Med Assoc 1999;215:1450-1454

2. Levy MG, Gookin JL, Poore M, et al. Tritrichomonas foetus and not Pentatrichomonas hominis is the etiologic agent of feline trichomonal diarrhea. J Parasitol 2003;89:99-104. 
3. Gookin JL, Levy MG, Law JM, et al. Experimental infection of cats with Tritrichomonas foetus. Am J Vet Res 2001;62: $1690-1697$.

4. Romatowski J. Pentatrichomonas hominis infection in four kittens. J Am Vet Med Assoc 2000;216:1270-1272.

5. Gookin JL, Stebbins ME, Hunt E, et al. Prevalence of and risk factors for feline Tritrichomonas foetus and Giardia infection. J Clin Microbiol 2004;42:2707-2710.

6. Romatowski J. An uncommon protozoan parasite (Pentatrichomonas hominis) associated with colitis in three cats. Feline Pract 1996;24:10-14.

7. Foster DM, Gookin JL, Poore MF, et al. Outcome of cats with diarrhea and Tritrichomonas foetus infection. J Am Vet Med Assoc 2004;225:888-892.

8. Kulda J. Trichomonads, hydrogenosomes and drug resistance. Int J Parasitol 1999;29:199-212.

9. Moreno SN, Mason RP, Muniz RP, et al. Generation of free radicals from metronidazole and other nitroimidazoles by Tritrichomonas foetus. J Biol Chem 1983;258:4051-4054.

10. Gookin JL, Foster DM, Poore MF, et al. Use of a commercially available culture system for diagnosis of Tritrichomonas foetus infection in cats. J Am Vet Med Assoc 2003;222: 1376-1379.

11. Gookin JL, Birkenheuer AJ, Breitschwerdt EB, et al. Singletube nested PCR for detection of Tritrichomonas foetus in feline feces. J Clin Microbiol 2002;40:4126-4130.

12. Vynckier LJ, Debackere M. Plasma ronidazole concentrations in sheep after intravenous, oral, intraruminal and intraabomasal administration. J Vet Pharmacol Ther 1993;16:70-78.

13. Baert L, Herman J, Remon JP. Influence of feeding on the bioavailability of ronidazole prolonged-release formulations in pigeons. J Vet Pharmacol Ther 1990;13:304-308.

14. Baert L, Vermeersch H, Remon JP. Effect of some organic vehicles on the rate of elimination of intravenously administered ronidazole in pigeons. J Vet Pharmacol Ther 1991;14:341-343.
15. Herman J, Vermeersch H, Remon JP, et al. Pharmacokinetics and bioavailability of ronidazole from a prolonged release tablet in the homing pigeon (Columba livia). J Vet Pharmacol Ther 1989;12:46-49.

16. Plumb D. Veterinary Drug Handbook, 2nd ed. Ames, IA: Iowa State University Press; 1995.

17. Oud JL, Reutlinger AH, Branger J. An investigation into the cytogenetic damage induced by the coccidiostatic agents amprolium, carbadox, dimetridazole and ronidazole. Mutat Res 1979;68:179-182.

18. Miwa GT, Wang R, Alvaro R, et al. The metabolic activation of ronidazole [(1-methyl-5-nitroimidazole-2-yl)-methyl carbamate] to reactive metabolites by mammalian, cecal bacterial and $T$ foetus enzymes. Biochem Pharmacol 1986;35:33-36.

19. Wolf FJ, Baylis FP, Smith GE, et al. Disposition of ronidazole by swine. 1. Radiocarbon content of tissues. J Agric Food Chem 1983;31:559-564.

20. Chen XG, Li J. Increasing the sensitivity of PCR detection in bovine preputial smegma spiked with Tritrichomonas foetus by the addition of agar and resin. Parasitol Res 2001;87:556-558.

21. Felleisen RS, Lambelet N, Bachmann P, et al. Detection of Tritrichomonas foetus by PCR and DNA enzyme immunoassay based on rRNA gene unit sequences. J Clin Microbiol 1998;36: 513-519.

22. Ho MS, Conrad PA, Conrad PJ, et al. Detection of bovine trichomoniasis with a specific DNA probe and PCR amplification system. J Clin Microbiol 1994;32:98-104.

23. Holland JL, Louie L, Simor AE, et al. PCR detection of Escherichia coli $0157: \mathrm{H} 7$ directly from stools: Evaluation of commercial extraction methods for purifying fecal DNA. J Clin Microbiol 2000;38:4108-4113.

24. Heininger A, Binder M, Ellinger A, et al. DNase pretreatment of master mix reagents improves the validity of universal 16S rRNA gene PCR results. J Clin Microbiol 2003;41:1763-1765. 